\title{
Reply to Raimondi et al.: Pediatric chest ultrasound versus conventional radiology: experimental evidence first
}

\author{
Paolo Tomà • Catherine M. Owens
}

Received: 13 February 2014 / Accepted: 19 March 2014 / Published online: 23 April 2014

(C) Springer-Verlag Berlin Heidelberg 2014

Sir,

We do appreciate the efforts of the authors in their reply [1] to our article [2].

We applaud them in their quest to develop ultrasound of the chest, and in doing so advertising their research for the first time in a journal of paediatric imaging.

We must point out, however, some limitations from analysis of their articles.

Artefacts, which are not directly convertible into images of the human body, and small foci of parenchymal echogenicity, along with abnormalities along the pleural surface of the lung, are used as biomarkers in an attempt to use direct comparison with the clinical signs.

The authors imply that US may be a more sensitive indicator of pathology than the chest radiograph (the current reference standard). We feel that this has not been proven in a rigorous scientific fashion and advise caution to the reader. It is also important to be mindful of the fact that the reference standard, i.e. the chest radiograph, must be performed with scrupulous attention to detail and technique optimised, and should be interpreted by experienced, well-trained readers.

\section{References}

1. Raimondi F, Cattarossi L, Copetti R (2014) Pediatric chest ultrasound versus conventional radiology: experimental evidence first. Pediatr Radiol. doi:10.1007/s00247-014-2930-3

2. Tomà $P$, Owens C (2013) Chest ultrasound in children: critical appraisal. Pediatr Radiol 43:1427-1434

P. Tomà $(\bowtie)$

Department of Imaging, Bambino Gesù Children's Hospital, IRCCS,

Piazza S. Onofrio, 00165 Rome, Italy

e-mail: paolo.toma@opbg.net

\section{M. Owens}

Department of Imaging,

Great Ormond Street Hospital for Children NHS Trust,

London, UK 